Korean J Ophthalmol 2020;34(3):262-264

https://doi.org/10.3341/kjo.2020.0010

\section{Rare Case of Isolated Superficial Angiomyxoma of the Eyelid}

\author{
Dear Editor,
}

Superficial angiomyxoma was first described in 1988 by Allen et al. [1] as a rare, benign, cutaneous tumor with a tendency for local recurrence after incomplete excision. Superficial angiomyxoma presents as cutaneous papules, nodules, or polypoid lesions in the trunk, head and neck region, and extremities. It often occurs in association with cardiac myxomas or as a form of Carney's complex, a rare, autosomal dominant disorder characterized by multiple cutaneous myxomas, mammary myxoma, cardiac myxoma and lentigines and endocrine overactivity [2]. However, isolated cases in the eyelid are very rare. To our knowledge, only three cases of isolated superficial angiomyxomas in the eyelid have been reported [3-5], and none of these cases were reported in Korea. Thus, we report the first case of isolated superficial angiomyxoma of the eyelid in Korea.

A 29-year-old woman visited our hospital with a painless round and movable soft subcutaneous mass at the left upper eyelid near the superolateral orbital rim (Fig. 1A). She first noticed the mass 6 months prior when she started to lose weight. The patient had undergone double eyelid blepharoplasty 9 years prior. She was otherwise healthy and on no medication. Visual acuity was $20 / 20$ in both eyes with correction, and the rest of the ophthalmic examination was unremarkable. Orbit computed tomography showed a 1.5 -cm-sized, ovoid soft tissue mass in the lateral aspect of the left upper eyelid (Fig. 1B). Under the impression of a dermoid cyst, orbital mass excision was performed. Intraoperative findings showed a mass that contained clear, adhesive, liquid material, loosely attached to a wide surface of the periosteum. The eyelid mass was excised completely (Fig. 1C). The histopathologic examina-

Received: February 7, 2020 Final revision: February 23, 2020

Accepted: February 26, 2020 tion revealed a $2.0-\mathrm{cm} \times 1.6-\mathrm{cm} \times 0.9-\mathrm{cm}$-sized myxoid tumor that was poorly circumscribed and extended into the surrounding fat tissue in a lobular growth pattern. The tumor had abundant thin-walled vessels in the background, which is a typical diagnostic feature of superficial angiomyxoma. The tumor cells were elongated and stellate or bipolar with indistinct cell borders; the tumor also had eosinophilic cytoplasm and oval hyperchromatic nuclei with inconspicuous nucleoli (Fig. 1D). The cellularity varied from mild to moderate, and mitotic figures were not observed. Immunohistochemically, tumor cells were negative for S-100 protein, smooth muscle actin, and estrogen receptors but were positive for CD34, consistent with superficial angiomyxoma (Fig. 1E). The patient did not complain of any cardiac symptoms, and routine electrocardiography showed a normal sinus rhythm. The patient also denied having any nevus or cutaneous lesion elsewhere in her body.

Superficial angiomyxoma is a rare, benign cutaneous myxoid tumor that often presents in middle age, although it may present at any age. Isolated eyelid angiomyxomas have previously been described to appear as multinodular [5], polypoid [3], or pedunculated [4], all of which involve the eyelid margin. However, they may also present as a smooth subcutaneous lump in the superotemporal quadrant of the eyelid, mimicking a dermoid or epidermoid cyst, as described in this case. Because there are no definitive clinical symptoms of superficial angiomyxoma, pathological findings are essential to correctly diagnose this disease. Histologically, superficial angiomyxoma usually involves the dermis with frequent diffuse extension into the subcutaneous tissue in a nodular pattern. A histopathology exam can show myxoid change with spindle- or stellate-shaped stromal cells that may be accompanied by thin-walled blood vessels but with no nuclear atypia or hyperchromasia. These spindle cells exhibit fibroblast features on microscopy, and they are negative for S-100 protein, smooth muscle actin, desmin, and keratin markers but positive for CD34 on immunostaining [1].

Superficial angiomyxomas of the eyelid are most frequently associated with Carney's complex and its subsets, NAME (nevi, atrial myxoma, myxoid neurofibroma, and ephelides) and LAMB (lentigines, atrial myxoma, muco- 

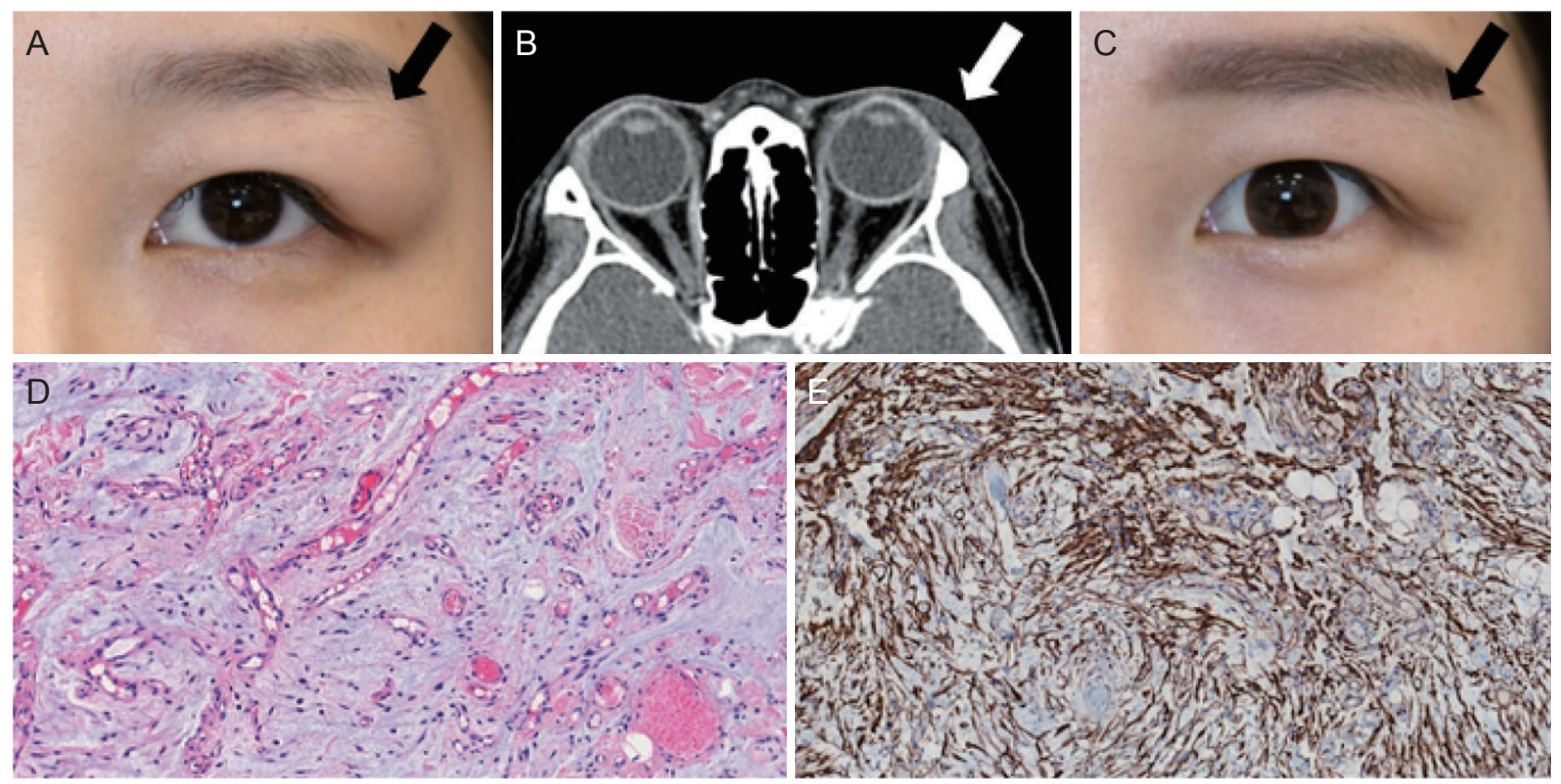

Fig. 1. Clinical and histologic ocular findings. (A) Photograph of the patient's eyelid at the first visit. A mass (arrow) at the left upper eyelid near the superolateral orbital rim was observed. (B) An orbital computed tomography image showed an ovoid soft tissue mass (arrow) in the lateral aspect of the left upper eyelid. (C) Photograph of the patient's eyelid after complete excision of the superficial angiomyxoma. The mass near the superolateral orbital rim had been removed (arrow). (D) Histopathologic analysis revealed a myxoid spindle cell tumor with elongated and stellate-shaped cells of mildly increased cellularity and nuclear polymorphism, surrounded by abundant thinwalled vessels $(\mathrm{H} \& \mathrm{E}, \times 200)$. (E) Immunohistochemical staining for CD34 showed strong positivity $(\times 10)$. Written informed consent was obtained from the patient.

cutaneous myxoma, and blue nevi) syndromes, and isolated eyelid angiomyxoma is extremely rare. Although systemic examination in our patient failed to demonstrate evidence of Carney's complex or other associated syndromes, multiple superficial angiomyxomas should always alert clinicians to the possibility of an underlying cardiac abnormality or Carney's complex. Superficial angiomyxomas have been reported to have a high local recurrence rate, up to $30 \%$ to $40 \%$, often as a result of incomplete excision [1]. Due to its possible association with Carney's complex and the likelihood of local recurrence, careful clinicopathological diagnosis and meticulous surgical excision are very important

\section{Young In Yun}

Department of Ophthalmology, Seoul National University Hospital, Seoul, Korea

\section{Kyu Sang Lee}

Department of Pathology, Seoul National University Bundang Hospital, Seongnam, Korea
Sang-In Khwarg

Department of Ophthalmology, Seoul National University Hospital, Seoul, Korea

\section{Namju Kim}

Department of Ophthalmology, Seoul National University Bundang Hospital, Seongnam, Korea

E-mail: kimnamju@snubh.org

\section{Conflict of Interest}

No potential conflict of interest relevant to this article was reported.

\section{References}

1. Allen PW, Dymock RB, MacCormac LB. Superficial angiomyxomas with and without epithelial components. Report of 30 tumors in 28 patients. Am J Surg Pathol 1988;12:51930 . 
2. Carney JA, Gordon H, Carpenter PC, et al. The complex of myxomas, spotty pigmentation, and endocrine overactivity. Medicine (Baltimore) 1985;64:270-83.

3. Yuen HK, Cheuk W, Luk FO, et al. Solitary superficial angiomyxoma in the eyelid. Am J Ophthalmol 2005;139:1141-2.
4. Ali N, Child CS, Michaelides M, Olver JM. Recurrence of a rare skin tumour: superficial angiomyxoma in the eyelid. Can J Ophthalmol 2011;46:205-6.

5. Pai V, Pai P, Mathew M. A rare case of isolated eyelid myxoma. J Pakistan Assoc Dermatol 2013;23:240-2. 